\title{
Reação de graduandos e pós-graduandos aos procedimentos instrucionais utilizados em disciplinas semipresenciais
}

\author{
Graduate and undergraduate students' reaction to the teaching procedures used in semipresential classes
}

Reacción de estudiantes de grado y posgrado a los procedimientos educativos utilizados en disciplinas semipresenciales

Henry Maia Peixoto ${ }^{1}$, Mariana Maia Peixoto², Elioenai Dornelles Alves ${ }^{3}$, Maria Cristina Soares Rodrigues ${ }^{4}$

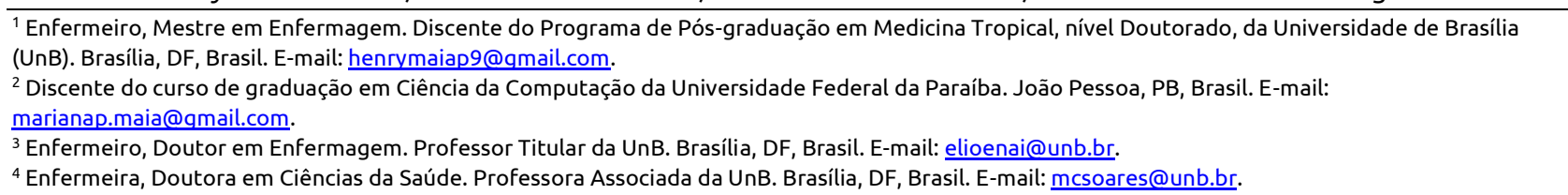

\section{RESUMO}

Este estudo teve por objetivo investigar as reações de graduandos e pós-graduandos aos procedimentos instrucionais utilizados em disciplinas semipresenciais da área de saúde. Pesquisa exploratória de abordagem quantitativa realizada em uma universidade pública em que participaram concluintes de disciplinas semipresenciais de graduação e pós-graduação, que versavam sobre educação e promoção da saúde. Entre os 19 procedimentos instrucionais avaliados, 15 (78,9\%) não apresentaram diferenças estatisticamente significantes entre os níveis acadêmicos. A maioria das variáveis, tanto entre graduandos (78,9\%) quanto entre pós-graduandos (89,5\%), apresentaram médias e medianas superiores a 7 em uma escala que variou de 0 (péssimo) a 10 (excelente). Portanto, pode-se concluir que ambos os grupos reagiram de forma semelhante aos procedimentos instrucionais e manifestaram opiniões satisfatórias sobre os mesmos. A compreensão sobre esses aspectos poderá subsidiar o desenho de disciplinas que utilizam procedimentos instrucionais adequados a alunos do ensino superior.

Descritores: Educação a Distância; Educação Superior; Aprendizagem; Avaliação Educacional.

\section{ABSTRACT}

The objective of this study was to investigate the reactions of undergraduate and graduate students to the teaching procedures used in semipresential classes. This exploratory study was performed with a quantitative approach at a public university, with undergraduate and graduate students who had completed semipresential classes on health promotion education. Among the 19 evaluated teaching procedures, 15 (78.9\%) did not show any statistically significant differences between the two academic levels. The means and medians for most variables, for both undergraduate (78.9\%) and graduate (89.5\%) students, were above 7 in a scale ranging between 0 (awful) and 10 (excellent). Therefore, it is concluded that both groups showed similar reactions to the teaching procedures and gave satisfactory opinions in this regard. Understanding these aspects can support designing class disciplines that use teaching procedures that are adequate to university students.

Descriptors: Education, Distance; Education, Higher; Learning; Educational Measurement.

\section{RESUMEN}

Se objetivó investigar las reacciones de estudiantes de grado y posgrado ante los procedimientos educativos utilizados en disciplinas semipresenciales del área de salud. Investigación exploratoria, de abordaje cuantitativo, realizado en universidad pública, en la que participaron alumnos acabando disciplinas semipresenciales de grado y posgrado, que trataban sobre educación y promoción de la salud. Entre los 19 procedimientos educativos evaluados, 15 (78,9\%) no presentaron diferencias estadísticamente significativas entre los niveles académicos. La mayoría de las variables, tanto entre estudiantes de grado (78,9\%) como de posgrado (89,5\%), presentaron promedios y medianas superiores a 7 , en una escala que varió de 0 (pésimo) a 10 (excelente). Consecuentemente, puede concluirse en que ambos grupos reaccionaron de manera semejante a los procedimientos educativos y manifestaron opiniones satisfactorias sobre ellos. La comprensión de tales aspectos podrá ayudar en el diseño de disciplinas que utilizan procedimientos educativos adecuados a alumnos de enseñanza superior.

Descriptores: Educación a Distancia; Educación Superior; Aprendizaje; Evaluación Educacional. 


\section{INTRODUÇÃO}

As tecnologias educacionais digitais estão sendo utilizadas nos cursos de graduação (GR) e de pósgraduação (PG) na área da saúde, tanto no Brasil como no exterior ${ }^{(1-2)}$. Tal fenômeno tem sido viabilizado pela expansão do acesso a internet, que, por meio das novas tecnologias da informação e comunicação (TICS), vem possibilitando o uso de ferramentas computacionais facilitadoras do processo ensino-aprendizagem estabelecido em disciplinas ofertadas totalmente à distância ou de forma semipresencial(3-6).

Assim, a educação a distancia (EaD) desafia e produz uma desacomodação nas práticas e concepções educacionais vigentes, criando novas formas de ensinar $e$ aprender adaptadas e potencializadas pelas $\operatorname{TICs}^{(7)}$, que, por sua vez, de maneira flexível, têm facilitado a interatividade, síncrona e assíncrona, ao incorporarem arquivos de sons, imagens e vídeos ao material didático ${ }^{(4,8-9)}$.

Neste sentido, o Núcleo de Estudos em Educação a Distância e Promoção da Saúde (NESPROM) da Universidade de Brasília (UnB) vem coordenando a oferta de disciplinas de graduação e pós-graduação, a partir de um modelo padrão de portfólios eletrônicos inseridos aos ambientes virtuais de aprendizagem, denominados Moodle-fólios, cuja utilização tem se constituído como pontos de encontros coletivos que permitem o registro dos processos e produtos resultantes das atividades desenvolvidas nas disciplinas ${ }^{(10)}$. A escolha do Moodle como ambiente virtual de aprendizagem justifica-se pelo fato de ser a plataforma de código aberto, livre e gratuito, mais utilizada na EaD(11). Na EaD, o ambiente de aprendizagem virtual reúne as ferramentas tecnológicas que serão utilizadas na mediação do processo de aprendizagem, por meio do bate-papo, dos materiais disponibilizados na biblioteca, do fórum de discussão, do texto colaborativo, entre outras possibilidades ${ }^{(1,8)}$.

Nesta perspectiva, a expansão da EaD precisa ser constantemente avaliada quanto à qualidade e eficácia das ações educativas propostas. Aliada a essa necessidade, a Avaliação Educacional vem se configurando como um campo em expansão no Brasil, considerada como prática que possibilita a transparência das realizações institucionais, tanto para a comunidade universitária, quanto para a sociedade. Portanto, há um crescente interesse em sistematizar a avaliação de cursos de graduação e de pós-graduação, a fim de viabilizar uma formação socialmente sustentada, bem como estabelecer o monitoramento das políticas dirigidas ao Ensino Superior ${ }^{(12)}$.

No entanto, estudos que avaliam sistematicamente os procedimentos instrucionais são $\operatorname{raros}^{(13)}$. Todavia, o Modelo de Avaliação do Impacto do Treinamento no Trabalho (IMPACT) merece destaque por ter sido utilizado para investigar diversas variáveis preditivas de eficiência e eficácia do treinamento, analisando a relação entre os níveis de reação, aprendizagem e impacto(14-15).

O modelo IMPACT destaca a importância de se conhecer a reação ou opinião do aluno sobre a programação, estabelecendo assim uma medida de satisfação que considera diversos aspectos das ações educacionais relativas ao desenvolvimento das atividades, à aplicabilidade e utilidade do treinamento, aos resultados, às expectativas de suporte organizacional e ao desempenho do instrutor ${ }^{(14,16)}$.

Dessa forma, o modelo teórico anteriormente citado demonstra que a reação ou satisfação dos participantes aos procedimentos instrucionais pode influenciar os resultados do evento educativo, uma vez que estes deveriam produzir reações favoráveis nos participantes.

Neste sentido, pesquisadores ${ }^{(13-14)}$ ao revisarem a literatura científica nacional, apresentam medidas de reação aos procedimentos instrucionais, específicas para cursos à distância, via internet, a saber: Reação aos Procedimentos Tradicionais: satisfação dos participantes com a qualidade dos objetivos de ensino, dos conteúdos, da sequência, das avaliações de aprendizagem, das estratégias e dos meios; Reação aos Procedimentos Web: satisfação dos participantes com a qualidade das ferramentas da web, tais como links, fóruns, banco de perguntas mais frequentes (FAQ), mural de notícias virtual, chats.

Pelo exposto, com o propósito de compreender características importantes relacionadas aos alunos inseridos na EaD, e se essas diferem de acordo com o nível acadêmico, na presente pesquisa objetivou-se analisar as reações aos procedimentos instrucionais na perspectiva de graduandos e de pós-graduandos matriculados em disciplinas semipresenciais da área da saúde, que utilizam portfólios eletrônicos como espaço pedagógico. 


\section{MÉTODOS}

Trata-se de um estudo do tipo exploratório, no qual foram utilizados dados quantitativos coletados no período de janeiro a fevereiro de 2011.

A pesquisa foi realizada, entre alunos da área de saúde, nas disciplinas de graduação, Promoção da Saúde 2 (PS2), Tópicos Avançados em Promoção da Saúde 1 (TAPS), Metodologia da Pesquisa em Saúde (MPS), no curso de Especialização em Educação e Promoção da Saúde (EEPS) e na disciplina Práticas Educativas em Ciências da Saúde (PECS) ofertadas pelos Programas de Pós-Graduação em Enfermagem e em Ciências da Saúde da Faculdade de Ciências da Saúde da Universidade de Brasília (UnB) e ministradas sob a coordenação do NESPROM.

O estudo foi submetido ao Comitê de Ética em Pesquisa da Faculdade de Ciências da Saúde da UnB e aprovado em dezembro de 2010, protocolo 137/10, com base na Resolução 196/96 do Conselho Nacional de Saúde que trata das diretrizes e normas regulamentadoras de pesquisa envolvendo seres humanos.

Os seguintes critérios de inclusão foram utilizados na amostra: todos os alunos de graduação, especialização, mestrado e doutorado, de ambos os sexos, devidamente matriculados, que concluíram as disciplinas ou o curso de especialização avaliado, no segundo semestre de 2010 e que aceitaram voluntariamente participar da pesquisa, mediante assinatura do Termo de Consentimento Livre e Esclarecido (TCLE).

Foram coletados dados primários por meio de um questionário, adaptados a partir de pesquisas nacionais que utilizaram o modelo IMPACT como norteador teórico(14,17), disponibilizado no ambiente virtual das disciplinas e do curso avaliado. O instrumento composto por duas partes, com a finalidade de coletar dados sociodemográficos (parte 1) e investigar as reações dos alunos aos procedimentos instrucionais (parte 2). A segunda parte utilizou uma escala do tipo Likert de 11 pontos, variando de zero (péssimo) a 10 (excelente), validada em outros estudos ${ }^{(13,15)}$.

Todos os alunos concluintes foram convidados a participar da pesquisa, todavia, dos 220 alunos concluintes, 148 (67,27\%) participaram efetivamente da pesquisa ao atenderem o convite respondendo ao questionário, caracterizando, portanto, uma amostragem por conveniência. As disciplinas TAPS, MPS e PS2 tiveram, respectivamente, $44,34 \%, 84,62 \%$ e $95,65 \%$ de seus alunos amostrados, o que representou $56,34 \%$ (80) do total de alunos de graduação concluintes. No curso de especialização EEPS e na disciplina PECS, dos alunos concluintes, respectivamente participaram da pesquisa $68 \%$ e $96,23 \%$, representando $87,18 \%$ do total de alunos de pós-graduação.

O estudo realizou análise de dados quantitativa aplicada aos dados pessoais dos alunos e às respostas numéricas dos participantes às escalas. Para composição do banco de dados, foram excluídos dados omissos e extremos univariados (escores $Z$ iguais ou superiores a 3,29 ou $+3,29$ ),

A análise estatística foi constituída por análise descritiva (medianas, médias, desvios padrões e percentagens), pelo cálculo da diferença entre médias que utilizou o teste não paramétrico de Mann-Whitney, depois de constatada a não normalidade das distribuições das frequências (Teste de Kolmogorov-Smirnov). Os testes de Qui-Quadrado e Exato de Fisher foram usados para verificar associações entre variáveis categóricas independentes. Foi adotado o nível de significância de $5 \%$. Para a execução das análises estatísticas, foi utilizado o Software Estatístico SPSS ${ }^{\circledR}$ (Statistical Package for Social Sciences), versão 17.0 .

\section{RESULTADOS}

\section{Características dos participantes}

Com relação à caracterização sociodemográfica, destaca-se que a amostra estudada foi constituída, preponderantemente, por 111 (75\%) estudantes do sexo feminino, sendo 56 (70\%) provenientes dos grupos constituídos por graduandos (GR) e 55 (80,9\%) por pósgraduandos (PG); solteiros predominaram em ambos os grupos, porém 28 (41,2\%) dos PGs e apenas 12 (15\%) dos GRs vivem com companheiro (a), o que representou uma diferença significativa $(p=0,001)$; quanto à idade dos envolvidos, observa-se uma diferença entre graduandos e pós-graduandos ( $p=0,001)$, havendo uma predominância no grupo GR da faixa etária entre 18 a 23 anos, 64 (80\%), e no grupo PG na faixa etária entre 24 a 34 anos, 34 (50\%). Não foi observado diferenças entre GR e PG nas variáveis: renda familiar e possuir computador, predominando renda familiar maior que oito salários mínimos em 99 
(66,9\%) dos alunos e possuir computador em 146 (98,6\%) dos estudantes.

\section{Procedimentos instrucionais}

As respostas dos 148 alunos participantes aos 19 itens do questionário que investigou as reações dos alunos aos procedimentos instrucionais, quando submetidas à análise exploratória foram identificados e excluídos 25 casos extremos univariados, que apresentaram valores referentes a escores $Z$ iguais ou superiores a $-3,29$ ou $+3,29$.

A Tabela 1 descreve média, desvio padrão (DP) e mediana do total de respostas dos alunos aos 19 procedimentos instrucionais avaliados.

Tabela 1: Resultado descritivo da escala de Reação aos Procedimentos Instrucionais. Brasília, DF, Brasil, $2012 . \mathrm{n=148.}$

\begin{tabular}{clcc}
\hline Itens & \multicolumn{1}{c}{ Variável } & Média \pm DP & Mediana \\
\hline 01 & Ligação entre o conteúdo proposto e os objetivos da disciplina & $8,49 \pm 1,45$ & 9 \\
02 & Ligação entre os objetivos da disciplina e os seus objetivos pessoais & $7,66 \pm 1,89$ \\
03 & Sequência de apresentação das oficinas/módulos & $8,14 \pm 1,74$ \\
04 & Sequência de apresentação dos momentos/fóruns & $8,21 \pm 1,64$ \\
05 & Linguagem utilizada no material da disciplina & $8,64 \pm 1,24$ \\
06 & Leituras recomendadas & $8,48 \pm 1,43$ \\
07 & Discussão no fórum do cafezinho & $6,24 \pm 2,83$ \\
08 & Discussão nas oficinas/módulos & $7,82 \pm 1,92$ & 9 \\
09 & Discussão nos chats & $6,00 \pm 2,92$ \\
10 & Novidades e lembretes divulgados no ambiente da disciplina (quadro de avisos) & $7,39 \pm 2,30$ \\
11 & Banco de perguntas mais frequentes sobre o curso/disciplina e suas respostas & $6,99 \pm 2,58$ \\
12 & Resposta apropriada às perguntas no fórum ajuda online & $7,20 \pm 2,68$ \\
13 & Links disponibilizados no ambiente eletrônico da disciplina & $8,30 \pm 1,55$ \\
14 & Atividades propostas ao final dos momentos/atividade das oficinas/módulos & $8,18 \pm 1,64$ \\
15 & Exercício de fixação (atividades obrigatórias) & $8,16 \pm 1,51$ \\
16 & Quantidade de conteúdo para a disciplina & 8 \\
17 & Quantidade de conteúdo para cada momento de cada oficina ou atividade de & 8 \\
18 & cada módulo & $8,16 \pm 1,57$ \\
19 & Quantidade de momentos por oficinas ou atividades por módulos da disciplina & $8,14 \pm 1,45$ & 8 \\
& módulo & $8,22 \pm 1,53$
\end{tabular}

A Tabela 2 apresenta os procedimentos de interação, apresentando média, desvio padrão (DP) e mediana de acordo como o nível acadêmico (GR e $P G$ ), além de analisar a diferença entre as médias apresentadas por graduandos e pós-graduandos por meio do Teste de Mann-Whitney, uma vez que todos os itens, de ambos os grupos, foram submetidos ao Teste de KolmogorovSmirnov, que constatou a não normalidade das distribuições das frequências. 
Tabela 2: Diferença entre grupos originados a partir da escala de

Reação aos Procedimentos Instrucionais. Brasília, DF, Brasil, 2012. n=148.

\begin{tabular}{|c|c|c|c|c|c|c|}
\hline Itens & Variável & $\begin{array}{c}\text { GR } \mathbf{n}=\mathbf{8 0} \\
\text { Média } \pm \text { DP }\end{array}$ & Mediana & $\begin{array}{c}\text { PG } n=60 \\
\text { Média } \pm \text { DP }\end{array}$ & Mediana & $\mathbf{p}$ \\
\hline 01 & $\begin{array}{l}\text { Ligação entre o conteúdo proposto e os } \\
\text { objetivos da disciplina }\end{array}$ & $8,19 \pm 1,64$ & 9 & $8,82 \pm 1,12$ & 9 & 0,03 \\
\hline 02 & $\begin{array}{l}\text { Ligação entre os objetivos da disciplina e os } \\
\text { seus objetivos pessoais }\end{array}$ & $7,47 \pm 1,95$ & 8 & $7,88 \pm 1,81$ & 8 & 0,22 \\
\hline 03 & $\begin{array}{l}\text { Sequência de apresentação das } \\
\text { oficinas/módulos }\end{array}$ & $8,00 \pm 1,95$ & 8 & $8,31 \pm 1,45$ & 8,5 & 0,60 \\
\hline 04 & $\begin{array}{l}\text { Sequência de apresentação dos } \\
\text { momentos/fóruns }\end{array}$ & $8,03 \pm 1,81$ & 8 & $8,43 \pm 1,42$ & 9 & 0,22 \\
\hline 05 & Linguagem utilizada no material da disciplina & $8,52 \pm 1,33$ & 9 & $8,78 \pm 1,13$ & 9 & 0,283 \\
\hline 06 & Leituras recomendadas & $8,24 \pm 1,50$ & 8 & $8,76 \pm 1,28$ & 9 & 0,026 \\
\hline 07 & Discussão no fórum do cafezinho & $6,53 \pm 2,79$ & 7 & $5,90 \pm 2,86$ & 7 & 0,140 \\
\hline 08 & Discussão nas oficinas/módulos & $7,58 \pm 1,92$ & 8 & $8,09 \pm 1,91$ & 9 & 0,047 \\
\hline 09 & Discussão nos chats & $5,93 \pm 2,93$ & 7 & $6,09 \pm 2,94$ & 7 & 0,666 \\
\hline 10 & $\begin{array}{l}\text { Novidades e lembretes divulgados no } \\
\text { ambiente da disciplina (quadro de avisos) }\end{array}$ & $7,06 \pm 2,49$ & 7,5 & $7,76 \pm 2,00$ & 8 & 0,105 \\
\hline 11 & $\begin{array}{l}\text { Banco de perguntas mais frequentes sobre o } \\
\text { curso/disciplina e suas respostas }\end{array}$ & $6,64 \pm 2,71$ & 7 & $7,40 \pm 2,36$ & 8 & 0,060 \\
\hline 12 & $\begin{array}{l}\text { Resposta apropriada às perguntas no fórum } \\
\text { ajuda on-line }\end{array}$ & $6,78 \pm 3,02$ & 8 & $7,69 \pm 2,14$ & 8 & 0,106 \\
\hline 13 & $\begin{array}{l}\text { Links disponibilizados no ambiente } \\
\text { eletrônico da disciplina }\end{array}$ & $8,03 \pm 1,71$ & 8 & $8,62 \pm 1,28$ & 9 & 0,046 \\
\hline 14 & $\begin{array}{l}\text { Atividades propostas ao final dos } \\
\text { momentos/atividade das oficinas/módulos }\end{array}$ & $7,82 \pm 1,83$ & 8 & $8,60 \pm 1,28$ & 9 & 0,009 \\
\hline 15 & Exercício de fixação (atividades obrigatórias) & $7,96 \pm 1,63$ & 8 & $8,40 \pm 1,33$ & 9 & 0,105 \\
\hline 16 & $\begin{array}{l}\text { Quantidade de conteúdo para a disciplina } \\
\text { Quantidade de conteúdo para cada }\end{array}$ & $7,96 \pm 1,80$ & 8 & $8,38 \pm 1,23$ & 8 & 0,300 \\
\hline 17 & $\begin{array}{l}\text { momento de cada oficina ou atividade de } \\
\text { cada módulo }\end{array}$ & $7,99 \pm 1,56$ & 8 & $8,31 \pm 1,31$ & 8 & 0,228 \\
\hline 18 & $\begin{array}{l}\text { Quantidade de momentos por oficinas ou } \\
\text { atividades por módulos da disciplina }\end{array}$ & $8,18 \pm 1,58$ & 8 & $8,26 \pm 1,47$ & 9 & 0,753 \\
\hline 19 & $\begin{array}{l}\text { Quantidade de horas de estudo sugerida } \\
\text { para cada momento das oficinas ou módulo }\end{array}$ & $8,08 \pm 1,62$ & 8 & $8,16 \pm 1,71$ & 8,5 & 0,597 \\
\hline
\end{tabular}

Os 19 itens descritos nas Tabelas 1 e 2 apresentaram as seguintes proporções: médias iguais ou superiores a sete foram identificadas em 16 itens (84,2\%) quando considerados todos os alunos, em 15 itens (78,9\%) quando considerado apenas o grupo GR e em 17 itens (89,5\%) quando considerado apenas o grupo PG; médias maiores ou iguais a cinco e inferiores a sete foram identificadas em apenas três itens $(15,8 \%)$ quando considerados todos os alunos, em quatro itens $(21,05 \%)$ quando considerado apenas o grupo GR e em dois (10,5\%) quando considerado apenas o grupo PG; médias inferiores a cinco não foram identificadas quando consideradas todos os alunos ou os grupos GR e PG. O DP apresentou o menor valor no item cinco $(1,24)$ e o maior valor no item nove $(2,92)$, quando considerados os dados advindos do total de alunos, nos itens cinco $(1,33)$ e 12 $(3,02)$ quando considerado apenas o grupo GR e nos itens um $(1,12)$ e nove $(2,94)$ quando considerado apenas o grupo PG. Portanto, neste instrumento os valores dos desvios padrões não foram considerados elevados na maioria dos itens, indicando uma pequena dispersão em torno das médias. Os itens apresentaram valores de mediana iguais ou superiores a sete em $100 \%$ dos alunos de graduação e pós-graduação.

Os procedimentos instrucionais que receberam as melhores avaliações foram: linguagem utilizada no material da disciplina (item 5), seguido por ligação entre o conteúdo proposto e os objetivos da disciplina (item 1) e leituras recomendadas (item 6). Por outro lado, os procedimentos instrucionais com piores avaliações, embora não tenha havido médias ou medianas inferiores ou iguais a cinco, foram: discussão nos chats (item 9), discussão no fórum do cafezinho (item 7) e banco de 
perguntas mais frequentes sobre o curso/disciplina e suas respostas (item 11).

Não foram verificadas diferenças significativas $(p<0,05)$ entre as médias apresentadas pelos grupos GR e PG em 14 itens da escala avaliada. Diferenças estatisticamente significante foram observadas apenas nos itens 1, 6, 8, 13 e 14 .

O item 1, ligação entre o conteúdo proposto e os objetivos da disciplina, apresentou valor de $p=0,03$, e um pequeno efeito $(d=0,46)$; o item 6 , leituras recomendadas, apresentou $p=0,026$, e efeito pequeno $(d=0,39)$; o item 8 , discussão nas oficinas/módulos, apresentou $p=0,047$ e um pequeno efeito $(d=0,27)$; o item 13 , links disponibilizados no ambiente eletrônico da disciplina, apresentou $p=0,046$ e um pequeno efeito $(d=0,39)$ e o item 14 , atividades propostas ao final dos momentos/atividades das oficinas/módulos, apresentou $p=0,009$ e um médio efeito $(d=0,50)$.

\section{DISCUSSÃO}

A tendência de gênero identificada na pesquisa, acompanha o perfil de estudantes universitários apresentado em um estudo ${ }^{(18)}$ que avaliou a inserção da mulher no Ensino Superior brasileiro, afirma que o número de mulheres supera o de homens em $12 \%$, sendo estas a maioria em $69 \%$ das áreas do conhecimento. Os autores demonstram, ainda, que a maioria dos estudantes de graduação brasileiros tem renda familiar entre três a 10 salários mínimos.

Estudo realizado em um curso para alunos de capacitação, destinado a gestores pedagógicos na área de saúde no NESPROM, identificou-se tendências semelhantes para gênero e faixa etárias ${ }^{(19)}$. Quanto ao acesso ao computador, uma pesquisa(20), ao avaliar características de estudantes de Enfermagem, identificou que $97 \%$ dos estudantes possuíam acesso a computador, o que, segundo o autor, demonstra o avanço da sociedade moderna e globalizada, em que os meios eletrônicos são essenciais ao desenvolvimento das suas funções.

No que diz respeito aos procedimentos instrucionais, estudos realizados no âmbito da $\operatorname{EaD}^{(14,17,21)}$ utilizaram instrumentos semelhantes ao adotado no presente estudo para avaliarem a reação aos procedimentos instrucionais entre alunos do ensino à distância. Estes, apresentaram, respectivamente, médias superiores a sete em $84,2 \%, 88,9 \%$ e $46,2 \%$ dos participantes, demonstrando que os valores identificados no presente estudo se assemelham aos encontrados nos dois primeiros estudos, indicando que os procedimentos instrucionais das disciplinas e do curso em questão foram bem avaliados por ambos os grupos. Em outro estudo(22), foi observado que a prática online foi bem recebida pelos alunos e este tipo de ensino é apropriado para fornecer conhecimento e oportunidade de desenvolver habilidades.

Assim, apesar de terem sido identificadas diferenças estatisticamente significantes entre os cinco itens descritos na Tabela 2, quatro apresentaram diferenças pequenas e uma moderada, indicando que alunos de GR e PG tiveram opiniões semelhantes sobre a qualidade dos procedimentos instrucionais.

Promover avaliações a fim de garantir qualidade e a efetividade de ações educativas à distância é fundamental. Dependendo dos resultados esperados, os métodos e atividades de aprendizagem podem variar (22). Entre os aspectos a serem avaliados destaca-se a reação dos alunos aos procedimentos instrucionais, uma vez que podem influenciar a consecução dos objetivos do processo educativo ${ }^{(13)}$. Em outro estudo ${ }^{(23)}$ aponta-se alguns indicadores de entrada para a avaliação de uma disciplina semipresencial, tais como: a qualidade da infraestrutura (ambiente virtual de aprendizagem, os recursos para comunicação e interação professor-alunotutor e os laboratórios de informática) e a qualidade do planejamento da disciplina (plano de ensino, material didático, bibliografia e mídias).

Em um trabalho, realizado com estudantes da área da saúde, foi observado relatos de uma experiência positiva com o curso online. Os alunos, em grande proporção, concordaram fortemente que o curso foi bem organizado, satisfez as suas expectativas em termos de conteúdo, custo, créditos e tempo(24). Uma revisão sistemática(25) concluiu que a EaD possui um alto grau de satisfação manifestado pelos participantes visto que torna possível despertar o interesse, facilitar a comunicação e criar um ambiente não ameaçador para que os alunos expressem suas opiniões.

Neste sentido, os resultados identificados na presente pesquisa indicam a satisfação dos alunos com a qualidade dos objetivos do ensino, dos conteúdos, da sequência, das estratégias e das ferramentas apresentadas no ambiente virtual de aprendizagem. 


\section{CONCLUSÃO}

Os resultados apresentados demonstram que tanto graduandos quanto pós-graduandos reagem de forma semelhante e satisfatória aos procedimentos instrucionais utilizados nas disciplinas semipresenciais avaliadas, indicando, portanto, a adequação, no âmbito do ensino superior da área de saúde, da proposta educativa baseada em portfolio eletrônico (Moodle-fólio), independentemente do nível acadêmico.

\section{REFERÊNCIAS}

1. Cogo ALP, Pedro ENR, Silva APSS, Valli GP, Specht AM. Tecnologias digitais no ensino de graduação em enfermagem: as possibilidades metodológicas por docentes. Rev. Eletr. Enf. [Internet]. 2011 [acesso em: $30 \mathrm{dez} 2013$ ];13(4):657-64. Disponível em: http://dx.doi.org/10.5216/ree.v13i4.12562. 2. Nunes TWN, Franco SRK, Silva VD. Como a educação a distância pode contribuir para uma prática integral em saúde? Rev Bras Educ Med [Internet]. 2010 [acesso em: 30 dez 2013];34(4):554-64. Disponível em: http://dx.doi.org/10.1590/S0100-55022010000400011. 3. Cogo ALP, Silveira DT, Pedro ENR, Tanaka RY, Catalan VM. Aprendizagem de sinais vitais utilizando objetos educacionais digitais: opinião de estudantes de enfermagem. Rev Gaucha Enferm [Internet]. 2010 [acesso em: $30 \mathrm{dez} 2013$ ];31(3):435-41. Disponivel em: http://dx.doi.org/10.1590/S198314472010000300005 .

4. Barbosa SFF, Marin HF. Web-based simulation: a tool for teaching critical care nursing. Rev Lat Am Enfermagem [Internet]. 2009 [acesso em: $30 \mathrm{dez} 2013$ ];17(1):7-13. Disponível em: http://dx.doi.org/10.1590/S0104-11692009000100002. 5. Peixoto HM, Peixoto MM, Alves ED. Aspects related to the permanence of undergraduate and graduate students in semipresential classes. Acta paul. enferm. [Internet]. 2012 [acesso em: 30 dez 2013];25(n. esp. 2):48-53. Disponível em: http://dx.doi.org/10.1590/S0103-21002012000900008. 6. Petit dit Dariel O, Wharrad H, Windle R. Exploring the underlying factors influencing e-learning adoption in nurse education. J Adv Nurs [Internet]. 2013 [acesso em: $30 \mathrm{dez}$ 2013];69(6):1289-300. Disponível em: http://dx.doi.org/10.1111/j.1365-2648.2012.06120.x. 7. Saraiva LM, Pernigotti JM, Barcia RM, Lapolli EM. Tensões que afetam os espaços de educação a distância. Psicol Estud [Internet]. 2006 [acesso em: $30 \mathrm{dez} 2013$ ];11(3):483-91. Disponivel em: http://dx.doi.org/10.1590/S141373722006000300004 .

8. Reed LF. Best practices for teachers new to distance education. Journal of Nursing Education and Practice [Internet]. 2013 [acesso em: $30 \mathrm{dez}$ 2013];3(8):92-8. Disponível em: http://dx.doi.org/10.5430/jnep.v3n8p92.

9. Peixoto HM, Peixoto MM, Alves ED. Learning strategies used by undergraduate and postgraduate students in hybrid courses in the area of health. Rev Lat Am Enfermagem [Internet]. 2012 [acesso em: 30 dez 2013];20(3):551-8. Disponível em: http://dx.doi.org/10.1590/S0104-11692012000300017. 10. Alves ED, Ribeiro LSN, Guimarães DCSM, Costa CMA, Peixoto $H M$, Martins EF, et al. Moodle-fólio para o ensino em saúde e enfermagem: avaliação do processo educacional. Rev. Eletr. Enf. [Internet]. 2012 [acesso em: $30 \mathrm{dez} 2013$ ];14(3):473-82. Disponível em: http://dx.doi.org/10.5216/ree.v14i3.19109.
Assim, no momento em que há um acelerado desenvolvimento das TICS, bem como sua crescente utilização na educação superior, a identificação de práticas pedagógicas, ancoradas em procedimento instrucionais facilitadores do processo ensinoaprendizagem, torna-se fundamental. Nesse sentido, a presente pesquisa poderá auxiliar no desenvolvimento e implementação de procedimentos instrucionais de novas disciplinas e/ou cursos na modalidade à distância, aperfeiçoando a qualidade da EaD na área da saúde.

11. Dias RA, Leite LS. Educação a distância: da legislação ao pedagógico. $1^{\text {a }}$ ed. Rio de Janeiro: Vozes; 2010. 12. Meira MDD, Kurcgant P. Avaliação de Curso de Graduação segundo egressos. Rev. esc. enferm. USP [Internet]. 2009 [acesso em: 20 dez 2013];43(2):481-5. Disponível em: http://dx.doi.org/10.1590/S0080-62342009000200031.

13. Zerbini T, Abbad G. Reação aos procedimentos instrucionais de um curso via internet: validação de uma escala. Estud. psicol. (Campinas) [Internet]. 2009 [acesso em: 20 dez 2013];26(3):36371. Disponivel em: http://dx.doi.org/10.1590/S0103166X2009000300009.

14. Zerbini T. Avaliação da transferência e treinamento em curso a distância [tese] [Internet]. Brasília: Instituto de Psicologia/UnB; 2007 [acesso em: 20 dez 2013]. Disponível em: http://repositorio.unb.br/handle/10482/3375.

15. Zerbini T, Abbad G. Impacto de treinamento no trabalho via internet. RAE electron. [Internet]. 2005 [acesso em: $20 \mathrm{dez}$ 2013];4(2). Disponível em: http://dx.doi.org/10.1590/S1676$\underline{56482005000200001}$.

16. Abbad GS. Um modelo integrado de avaliação do impacto do treinamento no trabalho - IMPACT [tese]. Brasília: Instituto de Psicologia/UnB; 1999.

17. Silva AP. Avaliação de uma disciplina semipresencial de graduação ofertada por meio da Internet na Universidade de Brasília [dissertação]. Brasília: Instituto de Psicologia/UnB; 2004. 18. Ristoff D, Tavares I, Melo HP, Leta J, Martins F, Lombardi $M R$, et al. Simpósio Gênero e Indicadores da Educação Superior Brasileira, Brasília-DF, 6 e 7 de dezembro de 2007 [Internet]. Brasília: Instituto Nacional de Estudos e Pesquisas Educacionais Anísio Teixeira; 2008 [acesso em: 20 dez 2013]. Disponível em: http://www.publicacoes.inep.gov.br/detalhes.asp?pub=4340. 19. Alves ED, Kusano MSE, Serpa MGN, Kusano LME, Quaresma M. Añejos y avances en la utilizacion de la internet para la enseãnanza superior de enfermería en el Brasil. Panamericana de Enfermería. 2004;1:12-16.

20. Pereira FJR, Santos SR, Silva CC. Caracterização de professores e estudantes de enfermagem em João Pessoa Paraíba. Cogitare Enferm [Internet]. 2010 [acesso em: $20 \mathrm{dez}$ 2013];15(3):486-91. Disponível em:

http://ojs.c3sl.ufpr.br/ojs/index.php/cogitare/article/view/1889 2.

21. Correa VP. Avaliação de treinamentos a distância em uma organização pública [dissertação] [Internet]. Brasília: Instituto de Psicologia/UnB; 2007 [acesso em: 20 dez 2013]. Disponível em: http://repositorio.unb.br/handle/10482/2964.

22. Edirippulige S, Smith AC, Armfield NR, Bensink M, Wootton R. Student perceptions of a hands-on practicum to supplement an online eHealth course. J Med Internet Res [Internet]. 2012 [acesso em: $20 \mathrm{dez} 2013] ; 14(6): \mathrm{e} 182$. Disponível em: http://dx.doi.org/10.2196/jmir.2029. 
23. Bertolin JCG, Marchi ACB. Instrumentos para avaliar disciplinas da modalidade semipresencial: uma proposta baseada em sistemas de indicadores. Avaliação (Campinas) [Internet]. 2010 [acesso em: $20 \mathrm{dez} 2013$ ];15(3):131-46.

Disponível em: http://dx.doi.org/10.1590/S1414-

40772010000300007.

24. Stark CM, Graham-Kiefer ML, Devine CM, Dollahite JS, Olson $\mathrm{CM}$. Online course increases nutrition professionals' knowledge, skills, and self-efficacy in using an ecological approach to prevent childhood obesity. J Nutr Educ Behav [Internet]. 2011 [acesso em: $20 \mathrm{dez}$ 2013];43(5):316-22. Disponível em: http://dx.doi.org/10.1016/j.jneb.2011.01.010.

25. Du S, Liu Z, Liu S, Yin H, Xu G, Zhang H, et al. Web-based distance learning for nurse education: a systematic review. Int Nurs Rev [Internet]. 2013 [acesso em: 30 dez 2013];60(2):16777. Disponivel em: http://dx.doi.org/10.1111/inr.12015.

Artigo recebido em 28/06/2012.

Aprovado para publicação em 12/08/2013.

Artigo publicado em 31/12/2013. 